\title{
Iba1+ cell density and morphology classification at postnatal day 10 in four hippocampal subregions of female and male C57BL/6J mice
}

\author{
Danielle Guez-Barber ${ }^{1}$, Max Wragan ${ }^{2}$, Dana Raphael ${ }^{2}$, \\ Haley M. Phillips ${ }^{2}$, Kira Lu², Sanghee Yun ${ }^{3,4}$, Amelia J. Eisch ${ }^{3,5 *}$
}

${ }^{1}$ Division of Neurology, The Children's Hospital of Philadelphia (CHOP), Philadelphia, PA, USA, 19104

${ }^{2}$ School of Arts and Sciences, University of Pennsylvania, Philadelphia, PA, USA, 19104

${ }^{3}$ Department of Anesthesiology and Critical Care Medicine, CHOP Research Institute, Philadelphia, PA, USA, 19104

${ }^{4}$ University of Pennsylvania Perelman School of Medicine, Philadelphia, PA, USA, 19104

${ }^{5}$ Department of Neuroscience, University of Pennsylvania Perelman School of Medicine, Philadelphia, PA, USA, 19104

*Correspondence: eischa@chop.edu

Keywords: microglia, macrophage, dentate gyrus, CA1, CA3, subregion

Pre-Submission Author Contributions (for details and explanations see $\underline{\text { CRediT)}}$ ):

Listed in alphabetical order by last name

Conceptualization: DGB, AJE, SY

Data curation: DGB, AJE, KLu, MW

Formal Analysis: AJE, SY

Funding acquisition: DGB, AJE

Investigation: DGB, KLu, HMP, DR, MW

Methodology: DGB, MW

Project administration: DGB, AJE

Resources: AJE

Software: --

Supervision: DGB, AJE, SY

Validation: HMP, DR

Visualization: DGB, AJE, DR, MW, SY

Writing, original draft: DGB, AJE, DR, MW

Writing, review \& editing: DGB, AJE, SY 


\section{ABSTRACT}

Microglia maintain normal brain function and support the brain's response to disease and injury. The hippocampus is an area of focus for microglial study due to its central role in numerous behavioral and cognitive functions. Interestingly, microglia and related cells in the hippocampus and throughout the brain are distinct in male vs. female rodents, even in early life. Indeed, postnatal day $(\mathrm{P})$-dependent sex differences in number, density, and morphology of microglia-like cells have been reported in certain hippocampal subregions. For example, P3 female mice have more phagocytic microglia in dentate gyrus (DG) molecular layer (Mol) and CA1-3 stratum oriens (SO) regions vs. male mice, while P8 - but not P15 - male rats have more volume immunoreactive for markers of microglia-like cells (Iba1 and CD68) in the CA1 stratum radiatum (SR) vs. female rats. In the mouse, $\mathrm{P} 10$ is roughly equivalent to human term gestation, making it a common timepoint to study for many translationally-relevant neurobiological processes. However, sex differences in hippocampal microglia have not been examined in the P10 mouse hippocampus. In addition, key subregions of the hippocampus - CA3 SR, DG hilus - have not yet been assessed for sex differences in microglia. To address these knowledge gaps, we quantified Iba1+ cell densities and classified lba1+ cell morphology in P10 male and female C57BL/6J mice. Four subregions in the bilateral anterior hippocampus were analyzed in 40-micrometer coronal sections: DG Mol (Mol), DG Hilus, CA1 SR and stratum lacunosum moleculare (CA1), and CA3 SR and stratum lucidum (CA3). Light microscope images (40x) were analyzed offline for Iba1+ cell density and morphology by an observer blind to sex. The morphology of each Iba1+ cell was used to place cells into one of four previously-published categories: Round or ameboid (round-ish soma, no processes), Stout (round-ish soma, short process), Thick (irregular soma with few, thick processes), or Thin (irregular soma with multiple thin processes). Analysis of Iba1+ cell density shows no difference between male and female mice in Mol, Hilus, CA3, or CA1 (male $n=6$, female $n=7$ ). However, morphology classification shows a sex-dependent difference in the Mol and Hilus, with female mice having a greater percentage of Thick Iba1+ cells vs. male mice (Mol, Hilus), and a lower percentage of Thin lba1+ cells vs. male mice (Mol). With our analysis, it is unclear whether this greater percentage of thick and lower percentage of thin Iba1+ cells in the female vs. male hippocampus means Iba1+ microglia in female mice are 'younger' or 'more active' than those in male mice. However, these data are important as they reveal sex differences in Iba1+ microglia in the P10 mouse hippocampus. We discuss these results in the context of the large literature on sex differences in rodent microglia in the early postnatal period. 


\section{INTRODUCTION}

Microglia are neuroimmune cells, but their functions go far beyond neuroimmunity. Microglia maintain normal brain function by clearing debris, pruning synapses, regulating neurogenesis, and even regulating cognition and mood-relevant behavior supported by brain regions like the hippocampus ${ }^{1-8}$. Microglia also support the brain's response to disease and injury ${ }^{1,9-11}$. Interestingly, microglia and related cells are distinct in male vs. female rodents, even in early life. In the hippocampus, a brain region whose development continues well into the postnatal period, there are sex-, postnatal day (P)-, and hippocampal subregion-dependent differences in number, density, and morphology of microglia-like cells. For example, P3 female mice have more phagocytic microglia in dentate gyrus (DG) molecular layer (Mol) and CA1-3 stratum oriens (SO) regions vs. male mice ${ }^{12}$. In contrast, at P8 - but not $\mathrm{P} 15$ - male rats have more volume immunoreactive for markers of microglia-like cells (Iba1 and CD68) in the CA1 stratum radiatum (SR) vs. female rats ${ }^{13}$. In the mouse, P10 is roughly equivalent to human term gestation ${ }^{14}$, making it a common timepoint to study for many translationally-relevant neurobiological processes. However, sex differences in hippocampal microglia have not been examined in the P10 mouse hippocampus. In addition, key subregions of the hippocampus - CA3 SR, DG hilus - have not yet been assessed for sex differences in microglia.

To address these knowledge gaps, we quantified lba1+ cell densities and classified lba1+ cell morphology in $\mathrm{P} 10$ male and female C57BL/6J mice. Four subregions in the bilateral anterior hippocampus were analyzed in $40 \mu \mathrm{m}$ coronal sections: DG Mol (Mol), DG hilus (Hil), CA1 SR and stratum lacunosum moleculare (CA1), and CA3 SR and stratum lucidum (CA3). Light microscope images (40x) were analyzed offline for Iba1 + cell density and morphology by an observer blind to sex. The morphology of each lba1+ cell was used to place cells into one of four previously-published categories: round or ameboid (round-ish soma, no processes), stout (round-ish soma, short process), thick (irregular soma with few, thick processes), or thin (irregular soma with multiple thin processes). These data are important as they examine sex differences in Iba1+ microglia in the mouse hippocampus at P10, an understudied but important postnatal timepoint.

\section{METHODS}

\subsection{Animals}

Pregnant multiparous adult female C57BL/6J mice ( 12 weeks old) were shipped from Jackson Laboratory (stock \#000664) on embryonic day 14 (E14). Dams were individually-housed in a HEPA-filtered, closed airflow vivarium rack (Lab Products Inc., Enviro-Gard ${ }^{\mathrm{TM}}$ III) under a normal 12:12-hour light-dark cycle (06:00 on, 18:00 off) with ad libitum access to food (standard rodent chow, Lab Diets 5015 \#0001328) and water. The AAALAC-accredited facility at the Children's Hospital of Philadelphia (CHOP) Research Institute is temperature- $\left(22^{\circ} \mathrm{C}\right)$ and humidity-controlled $(30-70 \%)$. Mouse pups were kept in the same cage as the dam and their littermates following birth. Pups from 4 litters from 4 separate dams were used for this P10 analysis (pups: 6 males, 7 females). Mice were weighed once prior to tissue collection at P10. All mice were cared for in compliance with protocols approved by the Institutional Animal Care and Use Committee at $\mathrm{CHOP}$ and guidelines established by the NIH's Guide for the Care and Use of Laboratory Animals. Our scientific reporting adheres to the ARRIVE 2.0 guidelines ${ }^{15}$.

\subsection{Brain collection and tissue preparation}

Mice were sexed prior to brain collection based on anogenital distance and gonadal appearance. Mice were euthanized on P10 via live decapitation using sterilized, surgical scissors in compliance with $\mathrm{NIH}$ guidelines for euthanasia of rodent neonates. Once extracted from the skull, brains were 
fixed for 24 hours (h) at room temperature (RT) in 4\% paraformaldehyde (PFA). Following two PFA changes (one/day), brains were cryoprotected via placement in $30 \%$ sucrose in $0.1 \mathrm{M}$ PBS. Forty $\mu \mathrm{m}$ coronal sections (series of 1:6) through the anterior hippocampus were collected on a freezing microtome (Leica SM $2000 \mathrm{R}$ ) and stored in $1 \times$ PBS with $0.01 \%$ sodium azide at $4^{\circ} \mathrm{C}$ until processing for immunohistochemistry (IHC).

\section{$2.3 \mathrm{IHC}$}

IHC was performed as previously described ${ }^{16,17}$. Brain sections were mounted onto charged slides (Thermo Fisher Scientific, \#12-550-15) and left to dry for at least $2 \mathrm{~h}$ prior to IHC. Sections underwent antigen retrieval $\left(0.01 \mathrm{M}\right.$ citric acid, $\left.\mathrm{pH} 6.0,100^{\circ} \mathrm{C}, 15 \mathrm{~min}\right)$ followed by $\mathrm{RT}$ washes in $1 \mathrm{xPBS}$; all subsequent steps also occurred at RT. Non-specific protein binding was blocked via incubation with $3 \%$ Normal Donkey Serum (NDS) and $0.3 \%$ TritonX-100 in 1xPBS for 60 minutes (min). Following pretreatment and blocking steps, sections were incubated overnight with rabbit-anti-lba1 (1:500; Wako Chemicals, \#019-19741) in a carrier of 3\% NDS and 0.3\% Tween-20 in 1xPBS. Eighteen hours later, sections were rinsed with $1 \times$ PBS prior to incubation with biotinylated donkey-anti-rabbit IgG (1:200; Jackson ImmunoResearch Laboratories, \#711-065-152) in 1.5\% NDS. After washing with 1xPBS, endogenous peroxidase activity was inhibited via incubation with $0.3 \%$ hydrogen peroxide in 1xPBS for 30 min. Following an additional set of 1xPBS washes, slides were incubated with an avidin-biotin complex (ABC Elite, Vector Laboratories, \#PK-6100) for $90 \mathrm{~min}$. Slides were washed with 1XPBS and immunoreactive cells were visualized via incubation with metal-enhanced diaminobenzidine (Thermo Fisher Scientific, \#34065) for 11-12 min. Slides were counterstained via Fast Red (Vector Laboratories, \#H3403), dehydrated in a series of increasing ethanol concentrations and Citrosolv, and coverslipped with DPX (Thermo Fisher Scientific, \#50-980-370).

\subsection{Image collection and preparation for analysis}

Images from immunostained sections were captured in bright-field light microscopy using an Olympus BX51 microscope with a 40x/0.9NA objective and an Olympus DP74 camera (widescreen aspect ratio, 16:10). For each mouse, a total of eight $400 x$ images were collected (4 hippocampal subregions from each hemisphere at the anterior hippocampus, $-2.05 \mathrm{~mm}$ from bregma), similar to prior work $^{18,19}$. Photomicrographs were collected by a person blinded to sex. Guidelines for taking photomicrographic images of the four anterior hippocampal subregions (dentate gyrus molecular layer [Mol], dentate gyrus hilus [Hil], CA3, CA1, Fig. 1) were established to enable anatomical matching of subregions across hemispheres and mice and to reduce inter-person variation in taking photomicrographs. These guidelines were as follows: Mol: the outer Mol near the DG crest was positioned in the bottom and lateral corner of the image. Hil: the DG crest was placed in the lateral and central screen such that the hilus extended into the middle of the image. $\underline{C A}$ : the image was taken lateral to the hippocampal fissure and suprapyramidal DG granule cell layer and medial and dorsal to the CA3 pyramidal cell layer. CA1: the hippocampal fissure above the suprapyramidal DG granule cell layer was positioned at the bottom of the image at a placement that was parallel to the CA1 pyramidal cell layer. After photomicrographs were collected, a region of interest (ROI) was drawn on each image using FIJI by an observer blinded to sex. This ROI ensured the appropriate regions would be analyzed (e.g. DG $\mathrm{Mol}$ ) and other regions that may be in the image would be excluded (e.g. DG granule cell layer), and enabled quantification of total pixels within each ROI. For analysis, ROI areas (in pixels) were converted to $\mathrm{um}^{2}$ based on 1 pixel $=0.0231 \mathrm{um}^{2}$.

\section{$2.5 \mathrm{lba} 1+$ cell density determination and morphology classification}

For both cell density and morphology data collection, assessment was exhaustive; that is, all Iba1+ cells in the ROI of each photomicrograph were evaluated. Criteria for inclusion as an Iba1+ cell were 
a consistently-labeled soma easily distinguishable from the counterstain and the presence of an entire cross-sectional view through the soma in the focal plane captured in the photomicrograph. The photomicrographs for each subregion were annotated to indicate each Iba1+ cell that was counted and its morphology classification. This enabled a permanent record of the data collection and also allowed data collection validation as additional observers of the original, non-annotated photomicrographs could compare their results with the annotated versions. For Iba1+ cell density determination (Fig. 2), 6 male and 7 female pups from 4 different litters and dams were examined. Iba1+ cell count for one hemisphere for each of the four hippocampal subregions (Mol, Hilus, CA3, CA1) was divided by the area of the ROI to generate an Iba1+ cell density for that hemisphere. Density values for each hemisphere were averaged such that each mouse was represented by one density value for each subregion. For Iba1+ cell morphology classification (Fig. 3), 3 male and 3 female pups from 3 different litters and dams were examined. The morphology of each lba $1+$ cell guided placement of each cell into one of four previously-published categories ${ }^{12,20-23}$ : Round or ameboid (round-ish soma, no processes), Stout (round-ish soma, short process), Thick (irregular soma with few, thick processes), or Thin (irregular soma with multiple thin processes). Number of cells in each category was transformed into a percentage based on the total number of Iba1+ cells classified. A percentage for each of the four categories (Round, Stout, Thick, and Thin) was generated for each hemisphere in each subregion. The four percentages from hemisphere subregions were averaged with the other hemisphere subregion percentage, such that each mouse subregion was represented by four category percentages.

\subsection{Data analysis and statistics}

Sex of the mice was masked until data collection was complete. Data were analyzed via Prism 9 (Graphpad). Group distribution was first assessed by the Shapiro-Wilk test. Normally-distributed Iba1+ cell density measures for each subregion were next tested via t-test for an effect of sex. Normally-distributed lba1+ cell morphology classification for each subregion was tested via 2-way ANOVA (main effects: Sex [Male, Female] X Morphology [Round, Stout, Thick, Thin]). Post-hoc analysis was performed via Bonferroni's multiple comparison test. Data are expressed as mean +/SEM and $\alpha=0.05$. Statistical results are provided in the figure legends; only a summary of analyses is provided in the Results. In figures, asterisks are used to indicate t-test, main effect, or interaction significance (e.g. ${ }^{* *} p<0.001,{ }^{* * * *} p<0.0001$ ), and italicized lowercase letters are used to indicate posthoc significance (e.g. a'p<0.01, a" $p<0.001$ ).

\section{RESULTS}

\section{1 lba1+ cell densities in four hippocampal subregions were not different between male and female} C57BL/6J mice at postnatal day 10 (P10). Iba1+ cells were evident in all four subregions (Mol, Hilus, CA3, CA1) of all male and female mice examined. Following the guidelines for selecting subregion areas to consistently sample across mice (Fig. 1), Iba1+ cell densities were quantified (Fig. 2). Iba1+ cell density was similar in male and female mice in the Mol, Hilus, CA3, and CA1 (unpaired t-test, p's>0.05).

\subsection{Classification of Iba1+ cell morphology in mouse P10 hippocampal subregions shows a} sex-dependent difference. In each subregion, Iba1+ Thick and Thin cells were evident, while Iba1+ Round and Stout cells were far more rare, and Round cells were sometimes absent (Fig. 2A-D). Analysis of percentage of Iba1+ cells in each morphology category revealed a sex-dependent effect. In the Mol (Fig. 3E), female mice had 34\% more Thick Iba1+ cells vs. male mice, but $29 \%$ fewer Thin Iba1+ cells vs. male mice. In the Hilus (Fig. 3F), female mice had $39 \%$ more Thick lba1+ cells vs. 
male mice. In CA3 (Fig. 3G), female mice had borderline ( $p=0.052) 24 \%$ fewer Thin lba1+ cells vs. male mice. In contrast to Mol, Hilus, and CA3, in CA1 there was no difference by sex in regard to Iba1+ cell morphology classification (Fig. $3 \mathrm{H}$ ).

\section{DISCUSSION}

Two findings are reported here: 1) There is no difference in Iba1+ cell density between male and female mice in four hippocampal subregions at P10: Mol, Hil, CA3, and CA1. 2) However, there is a sex-dependent difference in Iba1+ cell morphology classification in the P10 Mol and Hil; female mice have a greater percentage of Thick lba1+ cells vs. male mice (Mol, Hil) yet a lower percentage of Thin Iba1+ cells vs. male mice (Mol). The lack of sex-dependent difference in lba1+ cell density in the P10 hippocampus is interesting in regard to prior work which, while seminal in providing a timecourse of hippocampal microglia in the early postnatal mouse brain, did not consider the effect of sex or of hippocampal subregion ${ }^{18}$. In that work, measures of microglia in the entire anterior hippocampus of male mice steeply increased from P0 to P15, with the sharpest rise from P10 to P15. In other work in rats, male and female microglia differ in early development such that male mice have more total brain microglia at P4, but females have more microglia with an activated/amoeboid morphology at $\mathrm{P} 30-60^{20}$. Our work showing no sex-dependent difference in lba1+ cell density in four hippocampal subregions at P10 warrants additional analysis of both earlier and later timepoints in male and female mice as it may help identify when this "switch" occurs. Our morphology classification data are similarly interesting, particularly in that there is a greater percentage of Thick Iba1+ cells in the Mol and Hilus (and lower percentage of Thin lba1+ cells in the Mol) of female vs. male P10 mice. The meaning of these morphology classification categories ${ }^{12,20-22}$ is still debated. In the adult rodent, similar microglia morphology categories have been used as a gradient to reflect the degree of "activation" of microglia; Round microglia (ameboid, no processes) are considered to be relatively more active than the ramified and presumably surveilling Thin microglia. In contrast, in the early postnatal rodent, microglia morphology categories are proposed to reflect the degree of "age" or maturation of the microglia; Round microglia are considered relatively younger and less mature than the ramified Thin microglia. With our analysis, it is not possible to know whether the greater percentage of Thick and lower percentage of Thin Iba1+ cells in the female vs. male Mol means lba1+ microglia in female mice are "younger" or "more active" than those in male mice. Additional analysis with additional markers, for example to assess phagocytic activity, would help determine which of these interpretations is correct.

There are several limitations of this study. First, Iba1+ cells in this study are manually counted offline, thus restricting analysis of these three-dimensional cells to only two dimensions. Also, only two fields of view are assessed for each hippocampal subregion (left and right hemisphere). While the "gold standard" approach for quantifying P10 lba1 + cells is unbiased stereolog ${ }^{24-26}$ (a technique with which we have extensive experience ${ }^{16,17,27-30}$ ) the low variability of the data presented here suggest some merit of this cell density measurement approach; further validation is needed, ideally in parallel with stereology and ability to focus through the $Z$ plane. Second, we are assuming that Iba1+ cells are microglia. However, Iba1 is expressed in the brain in two cells with distinct functions ${ }^{31,32}$ : resident microglia and infiltrating peripheral macrophages. Although some of the lba1+ cells analyzed here may be infiltrating macrophages, prior research suggests this is a very low percentage ${ }^{20}$, particularly in the uninjured brain. Definitive assessment of the lba1+ cells analyzed in our work as microglia vs. infiltrating macrophages will require co-labeling with markers to distinguish microglia from infiltrating peripheral macrophages ${ }^{33}$. Third, the pups used for this study came from timed pregnant dams who were delivered to our facility during pregnancy. It remains to be seen if pups from dams bred in-house will have similar sex differences in morphology classification as presented here. Related and also 
unanswered questions are whether sex, litter, and litter size interact in a way to influence the results presented here.

In sum, here we show that while there is no difference in Iba1+ cell density between male and female mice in four hippocampal subregions at P10, there is a sex-dependent difference in Iba1+ cell morphology classification in the P10 Mol and Hil. Provision of these fundamental data from male and female mice at P10 - a rodent developmental time point translationally relevant to human term gestation $^{14}$ — fills a key knowledge gap. In addition, the data presented here from healthy P10 male and female mice will be useful for research on the role of microglia in injury models. For example, in mouse models relevant to hypoxia ischemia $(\mathrm{HI})$, sex differences in microglia after P10 $\mathrm{HI}$ are often presented without consideration of baseline microglia values ${ }^{34-38}$. Our demonstration of no sex difference in Iba1+ cell density - but a sex difference in lba1+ cell morphology classification - in P10 hippocampal subregions provides a baseline from which to interpret microglia changes seen after injury. 


\section{Funding and acknowledgements}

This work was supported by the Children's Hospital of Philadelphia (CHOP) Development Fund (AJE) and Bridge Funding (AJE). DGB is supported by "Remapping the Clinical Neurosciences through Translation and Innovation Training" (2T32NS091008; co-PIs: Jensen, Aguirre), an NINDS Institutional Postdoctoral T32 and by Supplemental Support for Clinicians to Pursue Research Training in Neuroscience from the CHOP Research Institute. HMP and MW were supported by the University of Pennsylvania's (Penn) Center for Undergraduate Research and Fellowships (CURF). DR was also supported by CURF as well as by funds from being named a Penn Department of Neuroscience Scholar. SY was supported by a 2020 PENN Undergraduate Research Foundation grant and a 2021 NASA HERO grant (80NSSC21K0814, PI: Yun). We are indebted to Sharon Xie for biostatistical guidance, F. Chris Bennett, Graham Peet and Mariko Bennett for microglia imaging advice and discussions, Margaret McCarthy for discussions on microglia and sex differences in early life, and Kelly Jordan-Sciutto's group for permission to use their imaging set-up in the initial stage of this project. Thanks to many Eisch Lab members and colleagues for helpful discussions and guidance, in particular Lyles R. Clark, Fred C. Kiffer, and Ruthie E. Wittenberg.

\section{Declaration of Interest}

None of the authors have any conflict of interest to declare.

\section{Data Availability Statement}

An electronic copy of all experimental data will be made available upon reasonable written request. 


\section{REFERENCES}

1. Sierra, A., Abiega, O., Shahraz, A. \& Neumann, H. Janus-faced microglia: beneficial and detrimental consequences of microglial phagocytosis. Front. Cell. Neurosci. 7, 6 (2013).

2. Hong, S., Dissing-Olesen, L. \& Stevens, B. New insights on the role of microglia in synaptic pruning in health and disease. Curr. Opin. Neurobiol. 36, 128-134 (2016).

3. Wu, Y., Dissing-Olesen, L., MacVicar, B. A. \& Stevens, B. Microglia: Dynamic Mediators of Synapse Development and Plasticity. Trends Immunol. 36, 605-613 (2015).

4. Lenz, K. M. \& Nelson, L. H. Microglia and Beyond: Innate Immune Cells As Regulators of Brain Development and Behavioral Function. Front. Immunol. 9, 698 (2018).

5. Tremblay, M.-Ė., Stevens, B., Sierra, A., Wake, H., Bessis, A. \& Nimmerjahn, A. The role of microglia in the healthy brain. J. Neurosci. 31, 16064-16069 (2011).

6. Sato, K. Effects of Microglia on Neurogenesis. Glia 63, 1394-1405 (2015).

7. Gemma, C. \& Bachstetter, A. D. The role of microglia in adult hippocampal neurogenesis. Front. Cell. Neurosci. 7, 229 (2013).

8. Nieto-Quero, A., Chaves-Peña, P., Santín, L. J., Pérez-Martín, M. \& Pedraza, C. Do changes in microglial status underlie neurogenesis impairments and depressive-like behaviours induced by psychological stress? A systematic review in animal models. Neurobiology of Stress 15, 100356 (2021).

9. Lyu, J., Jiang, X., Leak, R. K., Shi, Y., Hu, X. \& Chen, J. Microglial Responses to Brain Injury and Disease: Functional Diversity and New Opportunities. Transl. Stroke Res. (2020). doi:10.1007/s12975-020-00857-2

10. Rosi, S. A polarizing view on posttraumatic brain injury inflammatory response. Brain Circ 2, 126-128 (2016).

11. Salter, M. W. \& Stevens, B. Microglia emerge as central players in brain disease. Nat. Med. 23, 1018-1027 (2017).

12. Nelson, L. H., Warden, S. \& Lenz, K. M. Sex differences in microglial phagocytosis in the neonatal hippocampus. Brain Behav. Immun. 64, 11-22 (2017).

13. Weinhard, L., Neniskyte, U., Vadisiute, A., di Bartolomei, G., Aygün, N., Riviere, L., Zonfrillo, F., Dymecki, S. \& Gross, C. Sexual dimorphism of microglia and synapses during mouse postnatal development. Dev. Neurobiol. 78, 618-626 (2018).

14. Semple, B. D., Blomgren, K., Gimlin, K., Ferriero, D. M. \& Noble-Haeusslein, L. J. Brain development in rodents and humans: Identifying benchmarks of maturation and vulnerability to injury across species. Prog. Neurobiol. 106-107, 1-16 (2013).

15. du Sert, N. P., Hurst, V., Ahluwalia, A., Alam, S., Avey, M. T., Baker, M., Browne, W. J., Clark, A., Cuthill, I. C., Dirnagl, U., Emerson, M., Garner, P., Holgate, S. T., Howells, D. W., Karp, N. A., Lazic, S. E., Lidster, K., MacCallum, C. J., Macleod, M., Pearl, E. J., Petersen, O. H., Rawle, F., Reynolds, P., Rooney, K., Sena, E. S., Silberberg, S. D., Steckler, T. \& Wuerbel, H. The ARRIVE guidelines 2.0: updated guidelines for reporting animal research. BMJ Open Science 4, e100115 (2020).

16. Yun, S., Reynolds, R. P., Petrof, I., White, A., Rivera, P. D., Segev, A., Gibson, A. D., Suarez, M., DeSalle, M. J., Ito, N., Mukherjee, S., Richardson, D. R., Kang, C. E., Ahrens-Nicklas, R. C., Soler, I., Chetkovich, D. M., Kourrich, S., Coulter, D. A. \& Eisch, A. J. Stimulation of entorhinal cortex-dentate gyrus circuitry is antidepressive. Nat. Med. 24, 658-666 (2018).

17. Lagace, D. C., Donovan, M. H., DeCarolis, N. A., Farnbauch, L. A., Malhotra, S., Berton, O., Nestler, E. J., Krishnan, V. \& Eisch, A. J. Adult hippocampal neurogenesis is functionally important for stress-induced social avoidance. Proc. Natl. Acad. Sci. U. S. A. 107, 4436-4441 
(2010).

18. Kim, I., Mlsna, L. M., Yoon, S., Le, B., Yu, S., Xu, D. \& Koh, S. A postnatal peak in microglial development in the mouse hippocampus is correlated with heightened sensitivity to seizure triggers. Brain Behav. 5, e00403 (2015).

19. Lawrence, K. M., McGovern, P. E., Mejaddam, A., Rossidis, A. C., Baumgarten, H., Kim, A. G., Grinspan, J. B., Licht, D. J., Radaelli, E., Rychik, J., Peranteau, W. H., Davey, M. G., Flake, A. W. \& Gaynor, J. W. Prenatal hypoxemia alters microglial morphology in fetal sheep. J. Thorac. Cardiovasc. Surg. (2019). doi:10.1016/j.jtcvs.2019.06.102

20. Schwarz, J. M., Sholar, P. W. \& Bilbo, S. D. Sex differences in microglial colonization of the developing rat brain. J. Neurochem. 120, 948-963 (2012).

21. Gómez-González, B. \& Escobar, A. Prenatal stress alters microglial development and distribution in postnatal rat brain. Acta Neuropathol. 119, 303-315 (2010).

22. Osborne, B. F., Turano, A., Caulfield, J. I. \& Schwarz, J. M. Sex- and region-specific differences in microglia phenotype and characterization of the peripheral immune response following early-life infection in neonatal male and female rats. Neurosci. Lett. 692, 1-9 (2019).

23. Stence, N., Waite, M. \& Dailey, M. E. Dynamics of microglial activation: a confocal time-lapse analysis in hippocampal slices. Glia 33, 256-266 (2001).

24. Long, J. M., Kalehua, A. N., Muth, N. J., Hengemihle, J. M., Jucker, M., Calhoun, M. E., Ingram, D. K. \& Mouton, P. R. Stereological estimation of total microglia number in mouse hippocampus. J. Neurosci. Methods 84, 101-108 (1998).

25. Dalmau, I., Vela, J. M., González, B., Finsen, B. \& Castellano, B. Dynamics of microglia in the developing rat brain. J. Comp. Neurol. 458, 144-157 (2003).

26. VanRyzin, J. W., Marquardt, A. E., Argue, K. J., Vecchiarelli, H. A., Ashton, S. E., Arambula, S. E., Hill, M. N. \& McCarthy, M. M. Microglial Phagocytosis of Newborn Cells Is Induced by Endocannabinoids and Sculpts Sex Differences in Juvenile Rat Social Play. Neuron 102, 435-449.e6 (2019).

27. Clark, L. R., Yun, S., Acquah, N. K., Kumar, P. L., Metheny, H. E., Paixao, R. C. C., Cohen, A. S. \& Eisch, A. J. Mild traumatic brain injury induces transient, sequential increases in proliferation, neuroblasts/immature neurons, and cell survival: A time course study in the male mouse dentate gyrus. Front. Neurosci. 14, 612749 (2020).

28. Bulin, S. E., Simmons, S. J., Richardson, D. R., Latchney, S. E., Deutsch, H. M., Yun, S. \& Eisch, A. J. Indices of dentate gyrus neurogenesis are unaffected immediately after or following withdrawal from morphine self-administration compared to saline self-administering control male rats. Behav. Brain Res. 381, 112448 (2020).

29. Eisch, A. J., Barrot, M., Schad, C. A., Self, D. W. \& Nestler, E. J. Opiates inhibit neurogenesis in the adult rat hippocampus. Proc. Natl. Acad. Sci. U. S. A. 97, 7579-7584 (2000).

30. DeCarolis, N. A., Mechanic, M., Petrik, D., Carlton, A., Ables, J. L., Malhotra, S., Bachoo, R., Götz, M., Lagace, D. C. \& Eisch, A. J. In vivo contribution of nestin- and GLAST-lineage cells to adult hippocampal neurogenesis. Hippocampus 23, 708-719 (2013).

31. Zarruk, J. G., Greenhalgh, A. D. \& David, S. Microglia and macrophages differ in their inflammatory profile after permanent brain ischemia. Exp. Neurol. 301, 120-132 (2018).

32. Greenhalgh, A. D., Zarruk, J. G., Healy, L. M., Baskar Jesudasan, S. J., Jhelum, P., Salmon, C. K., Formanek, A., Russo, M. V., Antel, J. P., McGavern, D. B., McColl, B. W. \& David, S. Peripherally derived macrophages modulate microglial function to reduce inflammation after CNS injury. PLOS Biology 16, e2005264 (2018).

33. Bennett, M. L., Bennett, F. C., Liddelow, S. A., Ajami, B., Zamanian, J. L., Fernhoff, N. B., Mulinyawe, S. B., Bohlen, C. J., Adil, A., Tucker, A., Weissman, I. L., Chang, E. F., Li, G., Grant, G. A., Hayden Gephart, M. G. \& Barres, B. A. New tools for studying microglia in the mouse and 
human CNS. Proc. Natl. Acad. Sci. U. S. A. 113, E1738-46 (2016).

34. Mirza, M. A., Ritzel, R., Xu, Y., McCullough, L. D. \& Liu, F. Sexually dimorphic outcomes and inflammatory responses in hypoxic-ischemic encephalopathy. J. Neuroinflammation 12, 32 (2015).

35. Al Mamun, A., Yu, H., Romana, S. \& Liu, F. Inflammatory Responses are Sex Specific in Chronic Hypoxic-Ischemic Encephalopathy. Cell Transplant. 27, 1328-1339 (2018).

36. Tsuji, S., Di Martino, E., Mukai, T., Tsuji, S., Murakami, T., Harris, R. A., Blomgren, K. \& Åden, U. Aggravated brain injury after neonatal hypoxic ischemia in microglia-depleted mice. J. Neuroinflammation 17, 111 (2020).

37. Umekawa, T., Osman, A. M., Han, W., Ikeda, T. \& Blomgren, K. Resident microglia, rather than blood-derived macrophages, contribute to the earlier and more pronounced inflammatory reaction in the immature compared with the adult hippocampus after hypoxia-ischemia. Glia 63, 2220-2230 (2015).

38. Fernández-López, D., Faustino, J., Klibanov, A. L., Derugin, N., Blanchard, E., Simon, F., Leib, S. L. \& Vexler, Z. S. Microglial Cells Prevent Hemorrhage in Neonatal Focal Arterial Stroke. J. Neurosci. 36, 2881-2893 (2016). 


\section{FIGURES}

\section{Figure 1. Barber et al.}

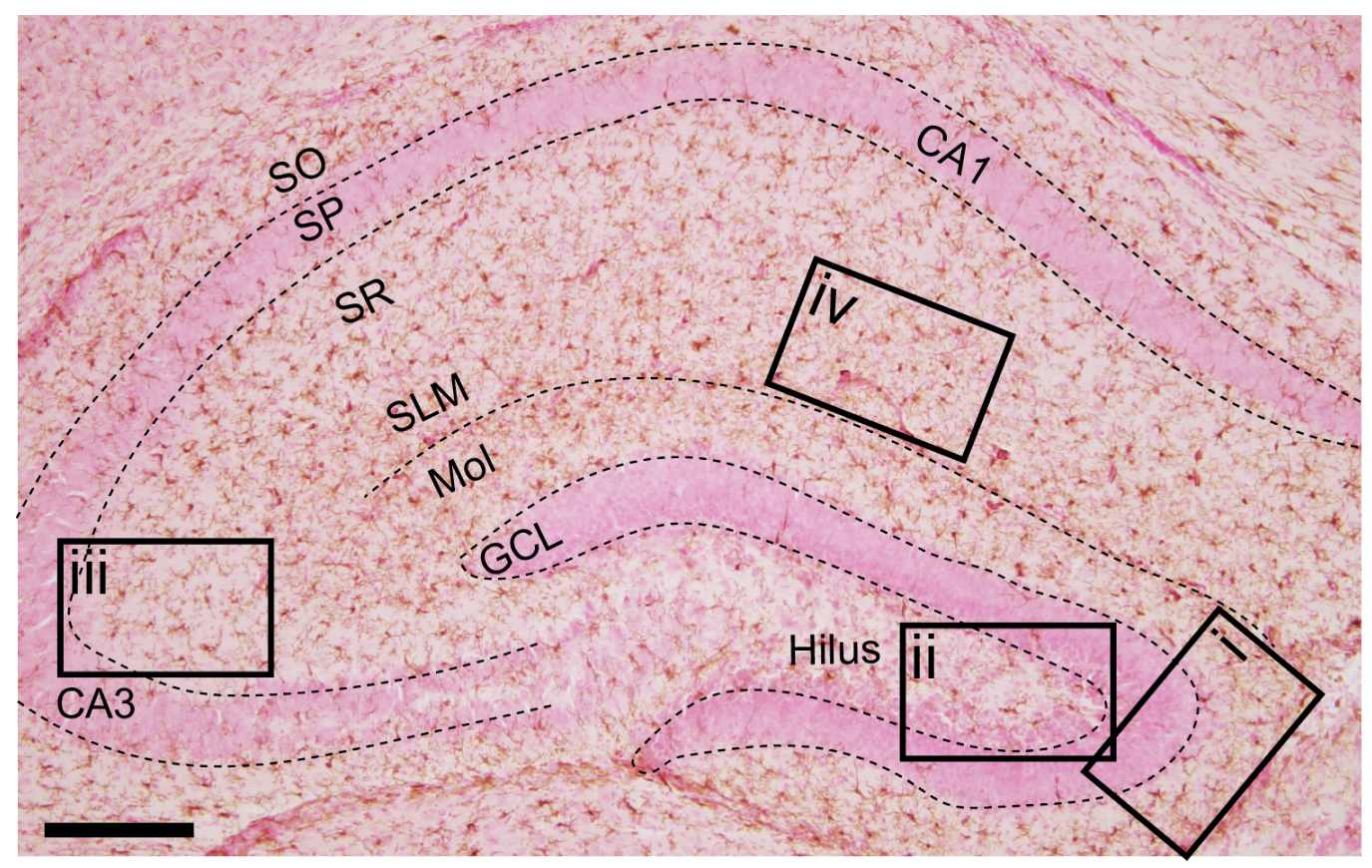

Figure 1. Hippocampal subregions analyzed for lba1+ cell density and morphology. This photomicrograph displays a P10 mouse hippocampal coronal brain section $(-2.05 \mathrm{~mm}$ from bregma) immunostained with an anti-lba1 antibody and counterstained with FastRed. Image was taken at 400x (objective: 40x, 0.9 NA). Principal cell layers of the dentate gyrus (DG; granule cell layer, GCL) and Ammon's horn (pyramidal cell layer or stratum pyramidal [SP] in CA1 and CA3) are darker pink and outlined with dashed lines. The hippocampal fissure that separates the DG molecular layer (Mol) from the CA1 stratum lacunosum moleculare (SLM) is shown as a single dashed line. The four outlined rectangles show the precise placement used to collect the data in this study: (i) DG Mol, (ii) DG Hilus, (iii) CA3 stratum radiatum (SR) and stratum lucidum (not shown), and (iv) CA1 SR and SLM. SO, stratum oriens. Scale bar=200um. 
Figure 2. Barber et al.
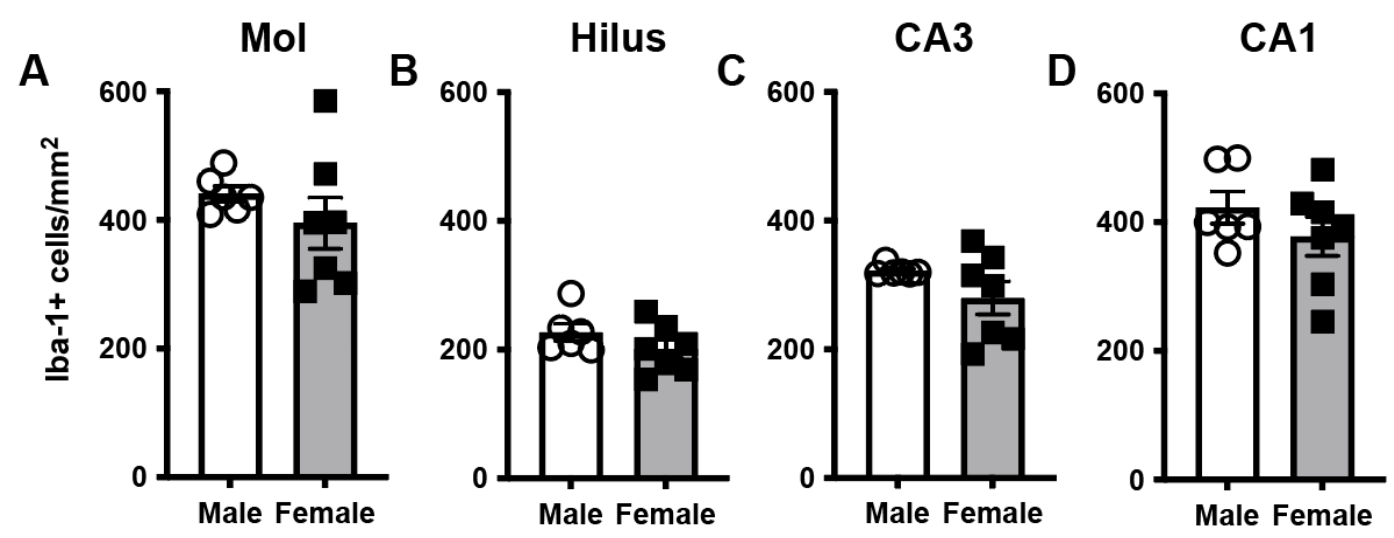

Figure 2. Iba1+ cell densities in four hippocampal subregions are not different between male and female C57BL/6J mice at postnatal day 10 (P10). Subregions analyzed are shown in Fig. 1. Subregions analyzed: (A) dentate gyrus molecular layer (Mol), (B) dentate gyrus hilus, (C) CA3 stratum radiatum and stratum lucidum (CA3), (D) CA1 stratum radiatum and stratum lacunosum moleculare (CA1). $Y$ axis in A applies to $B-D$. Male $n=6$, Female $n=7$. Unpaired t-tests, all p's $>0.05$. 
Figure 3. Barber et al.

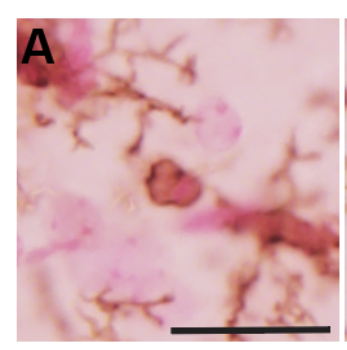

E<smiles>[V]=[Tl]</smiles>
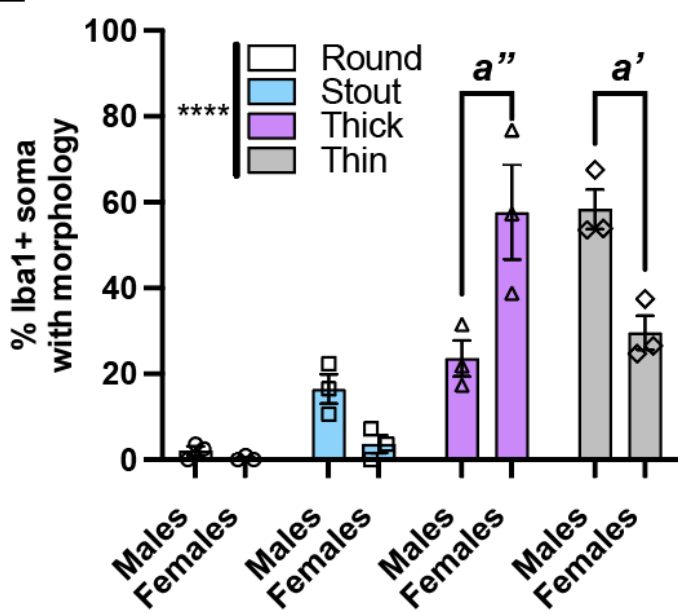

G

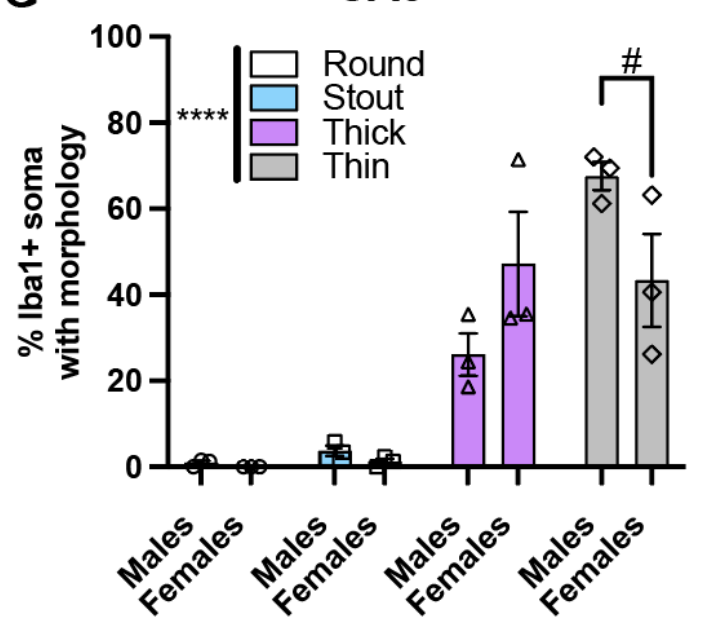

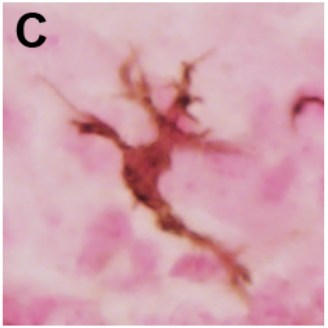

$\mathbf{F}$

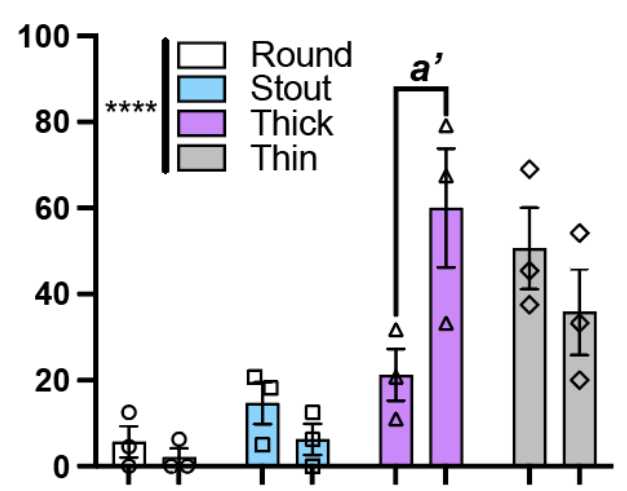

H

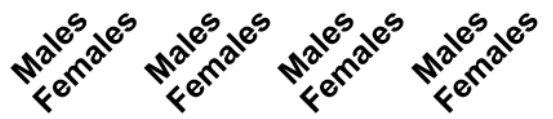

CA1
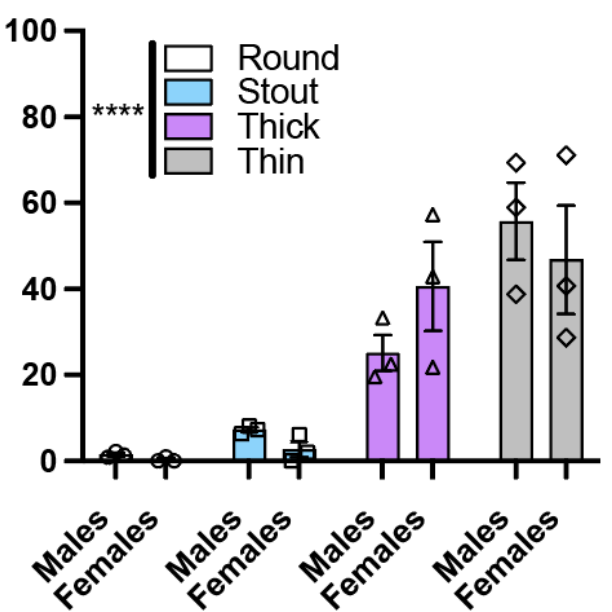

Figure 3. Classification of Iba1+ cell morphology in mouse P10 hippocampal subregions shows a sex-dependent difference. (A-D) Photomicrographs from the P10 hippocampus of Iba1+ cells that are representative of one of four morphological classifications: (A) Round or ameboid-like (no processes), (B) Stout (short, thick process), (C) Thick (soma with several thick processes), and (D) Thin (soma with several thin processes). Scale bar (A) = 25um and applies to (A-D). (E-H) Iba1+ cell morphology (\%) in male and female mice in each of four subregions of the anterior P10 hippocampus: molecular layer (Mol, E), hilus (F), CA3 (G), and CA1 (H). (E) In the Mol, two-way ANOVA (Sex X Morphology) revealed a main effect of Morphology $\left({ }^{* \star * *}: p<0.0001, F[3,16]=38.51\right.$ ) and an interaction of Sex X Morphology (not shown; $p<0.0001, F[3,16]=14.78$ ). Bonferroni posthoc 
test for multiple comparisons indicated female mice had a greater percentage of Thick Iba1+ Mol cells vs. male mice ( $a$ ": $p<0.001)$ and a lower percentage of Thin Iba1+ Mol cells vs. male mice (a': $p<0.005)$. (F) In the Hilus, two-way ANOVA revealed a main effect of Morphology $\left({ }^{* * * *}: p<0.0001, F\right.$ $[3,16]=14.04$ ) and an interaction of Sex X Morphology (not shown; $p<0.05, F[3,16]=5.045$ ).

Bonferroni posthoc test for multiple comparisons indicated female mice had a greater percentage of Thick Iba1+ Hilus cells vs. male mice (a”: p<0.001). (G) In CA3, two-way ANOVA revealed a main effect of Morphology $\left({ }^{* * *}: \mathrm{p}<0.0001, \mathrm{~F}(3,16)=38.48\right)$ and an interaction of Sex X Morphology (not shown; $p<0.05, F(3,16)=4.555)$. Bonferroni posthoc test for multiple comparisons indicated female mice had a borderline, but not significant, lower percentage of Thin Iba1+ CA3 cells vs. male mice (\#: $p=0.0052)$. (H) In CA1, two-way ANOVA revealed a main effect of Morphology ${ }^{* * * *}: p<0.0001, F[3$, $16]=24.85$ ) but no main effect of Sex and no interaction of Sex X Morphology (not shown; p's $>0.05$ ). Male $n=3$, Female $n=3$. 